\title{
Effects of signal intensity and reinforcer intensity on detection of electrical brain stimulation
}

\author{
B. J. HOMAN and J. W. KLING \\ Hunter Laboratory, Brown University, Providence, Rhode Island 02912
}

\begin{abstract}
The effects of intensity of signal and intensity of reinforcer on "yes/no" discrimination behavior were studied using electrical brain stimulation of one site for the signal and of another, contralateral site for the reinforcer. Accuracy declined as signal intensity declined, whereas accuracy was greatest with middle values of reinforcers. There was no statistical interaction between intensity of signal and intensity of reinforcer, indicating that processing of the aftereffects of the signals was not differentially influenced by intensity of reinforcers. Analysis of the detection behavior suggests that reinforcer level influenced decision criteria rather than sensitivity to the signal.
\end{abstract}

Brief trains of low-intensity electrical stimulation of hypothalamic and septal sites have readily discernible sensory properties: rats can be trained to indicate the presence or absence of such signals, and their accuracy of detection increases as the number of pulses or intensity of pulses in the signal is increased (Bass, 1974). The functional relations between accuracy of detection and signal intensity, and the partial temporal summation of intensity and number of pulses, is similar to results obtained with such exteroceptive stimuli as vision (Long, 1951) and audition (Garner, 1947) as well as those frequently observed for electrical stimulation of singleneuron preparations (e.g., Woodbury, 1965) or subcortical sensory nuclei (Gerken, 1970).

It is possible that the direct assessment of such sensory properties of low-intensity stimulation could contribute to the understanding of the neural mechanisms subserving the motivational and reinforcing effects of electrical stimulation of the brain (ESB). As in other studies of animal psychophysics where lengthy training and testing procedures are involved, it would be convenient in this assessment to employ ESB reinforcement. The advantages of such reinforcement over food reinforcement are that initial training is more rapid, position habits are less frequent and less persistent, and hundreds or thousands of trials may be run without satiation effects (Terman, 1970; Terman \& Kling, 1968).

If ESB is to be employed as reinforcement in the study of signal properties of weak electrical brain stimuli, there is the potential problem of interaction

Supported by Grant NS 12290 from the National Institute of Neurological and Communicative Disorders and Stroke. We thank Bonita J. Flück for the preparation of the brain sections. Reprints may be obtained from J. W. Kling, Hunter Laboratory, Brown University, Providence, Rhode Island 02912. between the two types of stimulation. Although preliminary observations (Bass, 1974) suggest that no such effects exist, there are a number of reasons for suspecting that some behavioral or physiological interactions might be found. For example, response biases of highly trained pigeons in visual detection tasks have varied with food-reinforcement probability (Wright, 1972), a finding in accordance with the expectations of modern signal detection theory (e.g., Swets, 1973). Similarly, small but consistent shifts in response bias in rats making auditory discriminations are produced when one or the other of the two classes of correct responses ("hits" or "correct rejections") are favored by manipulating the number of trains of reinforcing ESB (Hume, $1974 a, b)$. In addition to these effects of reinforcers on "steady state" behavior, an influence on the acquisition phase of brightness discrimination learning has been shown for pulse frequency (Keesey, 1966) and pulse-train duration (Lindholm \& Keesey, 1968) of ESB reinforcement. The possibility of physiological interactions between electrical signal stimuli and electrical reinforcing stimuli is suggested by experiments which demonstrate that ESB preceding or following the occurrence of the correct response in a training trial may enhance or reduce the effects of that trial, depending upon locus and timing of the stimulation or massing or spacing of trials (e.g., Fuster \& Uyeda, 1962; Mahut, 1964; Stein \& Chorover, 1968).

Because so many possibilities for interaction between signal and reinforcer seem possible, a systematic investigation of the effects of varying ESB reinforcement intensity on the detection of weak electrical brain signals was undertaken. An experimental design was employed which allowed the examination of accuracy of detection and potential response biases across a wide range of signal intensities and reinforcing stimulation intensities. 


\section{METHOD}

\section{Animals and Surgery}

Five adult male albino rats of the Charles River CD strain were used. They were maintained on ad-lib water and one daily feeding of $20 \mathrm{~g}$ Charles River rat diet. Each rat received stereotactic implantation of two chronic bipolar electrodes (Plastic Products MS 303-010) while anesthetized with a halothane and oxygen mixture. In each animal, one bipolar electrode was aimed at the lateral septal area, and the other at the lateral hypothalamus of the opposite hemisphere. Following completion of the experiment, animals were sacrificed with an overdose of sodium pentobarbital and perfused with physiological saline and a $10 \%$ neutral buffered Formalin solution. The brains were embedded in celloidin, sectioned at $40 \mu \mathrm{m}$, and stained with cresyl violet. Electrode loci were determined with the aid of the atlas of König and Klippel (1963), and are given in Table 1.

\section{Apparatus}

The experimental chamber $(24 \times 33 \times 48 \mathrm{~cm}$ high $)$ had three clear acrylic walls, an aluminum front panel, and a stainless steel grid floor. Three Gerbrands pigeon keys were mounted $3.8 \mathrm{~cm}$ above the floor and equally spaced in the front panel. Each key could be independently illuminated from behind. The chamber was housed in a light-tight, sound-attenuating, ventilated enclosure. A four-channel mercury swivel commutator (Berkley \& Kling, 1967) was attached to the ceiling of the enclosure. White noise (75 $\mathrm{dB}$ re $20 \mu \mathrm{N} / \mathrm{m}^{2}$ ) was continuously present in the enclosure. Recording and programming apparatus was located in an adjacent room.

Electrical brain stimulation consisted of symmetrical biphasic trains of rectangular pulses with the following parameters: frequency $=100$ pulses $/ \mathrm{sec} ;$ pulse width $=0.2 \mathrm{msec} ;$ pulse train $=0.48 \mathrm{sec}$. Stimuli for signal and for reinforcement purposes were generated by separate constant current systems, isolated from ground and from each other to avoid possible coupling artifacts. Each source was calibrated with a Keithley Model 160 digital multimeter, and monitored occasionally with Tektronix 502A CROs. At other times, CROs were disconnected from the circuits to avoid possible coupling of stimulators through the CROs. No ac sources were allowed in the chamber.

Following a postoperative period of at least 14 days, the animals were tested for reinforcing effects at each electrode site by attempting to shape barpressing in an apparatus with a single bar. Only animals which self-stimulated at both electrodes were included in this experiment. The range of self-stimulation values determined in this apparatus served as a guide for later training in the experimental apparatus.

\section{Procedure}

Preliminary training. For each rat, one bipolar electrode was

Table 1

Electrode Locus

König and Klippel Coordinates

\begin{tabular}{clrrrl}
\hline Rat & $\begin{array}{l}\text { Hemis- } \\
\text { phere }\end{array}$ & fig & $\mathrm{dv}$ & $\mathrm{ml}$ & Probable Structure \\
\hline \multirow{2}{*}{ 3B4 } & right & 14 & +0.4 & 0.2 & medial septum \\
& left & 31 & -2.2 & 1.4 & lateral hypothalamus \\
3B5 & right & 15 & +0.8 & 0.4 & lateral septum \\
& left & 33 & -2.4 & 1.6 & lateral hypothalamus \\
3B6 & right & 16 & +0.8 & 0.6 & lateral septum \\
& left & 33 & -2.4 & 1.4 & lateral hypothalamus \\
3B7 & right & 15 & +0.8 & 0.6 & lateral septum \\
& left & 32 & -2.0 & 1.0 & zona incerta \\
3B8 & right & 14 & +0.4 & 0.0 & medial septum \\
& left & 36 & -1.8 & 1.2 & Forel's Field $\mathrm{H}_{1}$ \\
\hline
\end{tabular}

arbitrarily selected for reinforcement purposes and the animal was allowed to self-stimulate for at least $4 \mathrm{~h}$ in the experimental apparatus. Both side keys were continually illuminated, and a press of either delivered one $0.48-\mathrm{sec}$ train of stimulation. Intensity of stimulation was varied to determine the effective range for each animal and to avoid excessive exposure to any one intensity.

Animals were then trained in the self-paced trials procedure by turning off the side-key lights and turning on the center-key light. Without further shaping, animals would press the center key which resulted in that light going out and both side-key lights coming on. As soon as either side key was pressed, one train of stimulation was delivered and the other side-key light was simultaneously extinguished. The selected side key remained illuminated until a total of three reinforced presses occurred, when it went dark. After a 1.5-sec intertrial interval, the center light again went on and the sequence repeated. This training continued until consistent self-paced responding occurred, with reinforcement intensities again being varied during sessions.

Discrimination training. In this and all subsequent sessions, the animal was connected to both stimulator circuits. When the illuminated center key was pressed, the light went off and on a random $50 \%$ of trials a 0.48 -sec train of stimulation was delivered to the signal electrode. Pressing the center key also initiated a delay of $0.5 \mathrm{sec}$ (to preclude possible overlap of signal and reinforcer), after which the side lights went on and the side keys were activated. If neither side key was pressed within $20 \mathrm{sec}$, all lights went out, the 1.5-sec intertrial interval began, and then the sequence started again. If the signal had been presented, three reinforced presses were allowed on the right key; if no signal had been presented, three reinforced presses were allowed on the left key. Thus, pressing the right key indicated a "yes" response, while pressing the left key indicated a "no-signal" response. The other two alternatives (pressing right key following no signal, or pressing left key after signal) were errors (a "false alarm" or a "miss"), which immediately resulted in a 5 -sec time-out, followed by the intertrial interval and repetition of the sequence.

Initially, the intensity of the signal was set high for each rat and the reinforcement intensity set in the middle of his range. Sessions continued until correct detections of the signal at or above $90 \%$ occurred for several blocks of 100 trials, when the signal intensity was lowered. This procedure was repeated until accuracy across signal intensities was stable for at least 2,000 trials. From these data, three signal intensities were selected for each rat, chosen so that detection accuracy was in the range of $90 \%-100 \%$ correct, $80 \%-90 \%$ correct, and $70 \%-80 \%$ correct. For each rat, three reinforcing intensities were chosen, covering a range from the lowest value that would support consistent responding to the highest that could be used without producing either strong motor effects or signs of aversions (e.g., jumping, vocalizing).

Testing procedure. Each subject ran through a sequence of nine blocks of 100 trials each day. Since descending intensity series have been found to provide more stable results than ascending series in similar testing situations (Bass, 1974; Blough, 1971; Terman, 1970), signal intensity was changed from high to middle to low after every block of 100 trials. Reinforcement intensity was changed after every $\mathbf{3 0 0}$ trials according to a preselected, counterbalanced sequence which was different for each animal. Each block of 100 trials was preceded by five warm-up trials. Following each block, the apparatus was momentarily shut off while intensity settings were changed. The signal and reinforcing intensities for each animal are presented in Table 2.

\section{RESULTS}

No significant difference attributable to the site of signal electrodes or the site of reinforcing elec- 
Table 2

Testing Intensities (in Microamps)

\begin{tabular}{llrlrc}
\hline & \multicolumn{2}{c}{ Signal } & & \multicolumn{2}{c}{ Reinforcement } \\
\cline { 2 - 3 } \cline { 5 - 6 } Rat & \multicolumn{1}{c}{ Site } & Intensities & & Site & Intensities \\
\hline 3B4 & septal & $94,74,60$ & & hypothalamus & $925,400,193$ \\
3B5 & hypothalamus & $54,35,29$ & & septal & $335,231,156$ \\
3B6 & hypothalamus & $122,91,64$ & & septal & $829,510,264$ \\
3B7 & septal & $35,25,19$ & & hypothalamus & $829,575,335$ \\
3B8 & hypothalamus & $103,64,49$ & septal & $988,608,400$ \\
\hline
\end{tabular}

trodes were found. Nor were any systematic differences obtained attributable to intensity of reinforcing stimulus used during training sessions relative to the intensities of the reinforcers used during testing. Accordingly, data have been pooled across subjects in the remaining analyses. For each animal, the percent correct responses was computed for each block of 100 trials; from these scores, the mean percent correct was obtained for the various combinations of signal and reinforcer intensities and for analysis of sequential effects.

Accuracy of signal detection increased as signal intensity increased. Analysis of variance indicated a significant main effect for signal intensity $(p<.01)$, and post hoc analyses revealed significant differences $(p<.01)$ between every pair of signal levels when averaging across reinforcement levels. For reinforcer intensity, analysis of variance revealed a significant main effect $(p<.05)$, with the greatest accuracy of detection occurring when the middle level of reinforcer was employed and the overall poorest detection accuracy occurring when the highest reinforcer level was employed $(p<.05)$. There was no

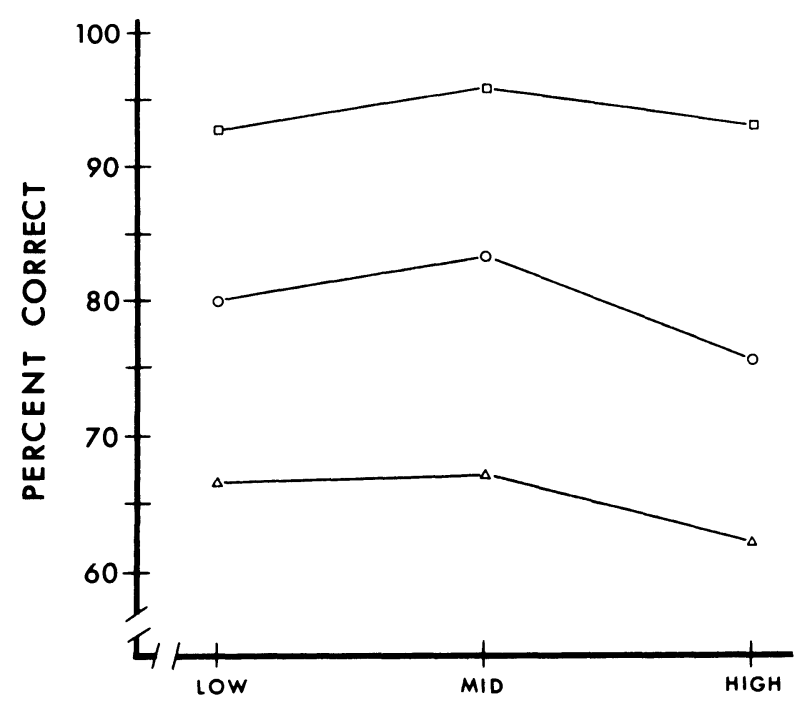

REINFORCER INTENSITY

Figure 1. Mean percent correct responses at each discriminative stimulus intensity as a function of reinforcing stimulus intensity. Signal intensity values indicated by square $=$ high, circle $=$ middle, and triangle $=$ low. significant interaction of signal and reinforcer intensities, nor did accuracy scores reveal any significant interactions involving sessions or sequences of intensities. Figure 1 summarizes these results as averaged across animals and test sessions.

Analysis of the sources of errors indicated that a "miss" (i.e., responding "no" when a signal had been presented) and a "false alarm" (i.e., responding "yes" when the signal had not been presented) were not equally likely to occur under all conditions. A significant $(p<.01)$ interaction between type of error and signal level was found. At high signal levels, the probability of a false alarm was slightly greater than the probability of a miss; at middle and low signal levels, the probability of a miss was significantly $(p<.01)$ greater than the probability of a false alarm. Reinforcer level influenced type of error only to the extent that the greatest probability of a false alarm generally occurred at the high reinforcer value $(p<.05)$. These results are summarized in Figure 2 . Note that the negative diagonal represents unbiased responding at each level of accuracy; data points lying above the diagonal reflect a bias toward responding "yes," while points below the diagonal represent a "no" bias.

These group data reflect well the behavior of the individual animals. At eight of the nine data points, all five animals had biases of the type shown by the group data. The only exception was shown by two animals that had very slight "no" biases in the high-signal/high-reinforcer condition when the other three animals were very slightly biased toward false alarms. Bias of any type was, of course, small under high-signal conditions.

The net time to complete each block of 100 trials (total time less time-outs for errors) was examined. Missing scores precluded analysis of times for two subjects. The overall pattern showed that trials were run off at a slower rate for the low level of reinforcement than for the middle or high level $(p<.05)$. Signal level had no effect on speed, nor was there a discernible Signal Intensity by Reinforcer Intensity interaction. A significant $(\mathrm{p}<.05)$ Sessions by Reinforcement Level interaction was found, attributable to progressively slower running at the low reinforcement level across daily sessions. Sequential effects within sessions were nonsignificant. 


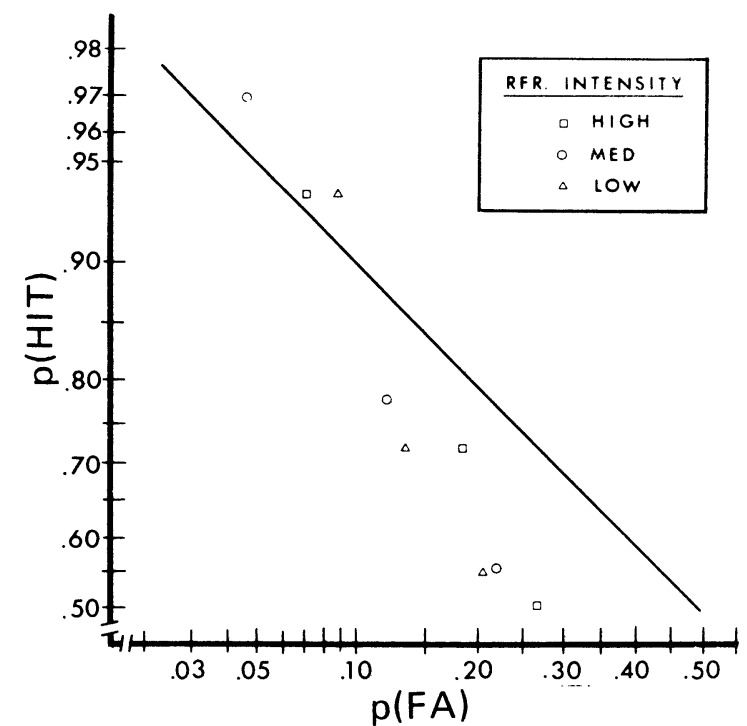

Figure 2. Mean probability of a response on signal trials (a hit) and on no-signal trials (a false alarm) at each signal intensityreinforcer intensity combination. Straight line indicates hypothetical unbiased behavior.

\section{DISCUSSION}

The primary purpose of the present investigation was to determine whether the detection of weak electrical brain signals was influenced by the intensity of the reinforcing stimulus used to maintain the behavior, and whether any interaction between these two sources of stimulation occurred. It was found that (a) accuracy of detection was positively correlated with intensity of signal stimulation; (b) across all levels of signal intensity, the medium level of reinforcer gave the best accuracy of detection; and (c) there was no significant degree of statistical interaction between signal intensity and reinforcer intensity.

In addition to prac :al implications for studies of animal psychophysics, this latter finding seems to put some interesting constraints on the models of electrical brain stimulation detection and reinforcement which might be developed. In the present study, all electrodes, whether used for signal or for reinforcement purposes, were shown to be positively reinforcing before the discrimination training was begun. If the brain had a single reinforcement system (Olds \& Olds, 1965), activation of all our electrodes might be expected to produce activity in that system. Neural aftereffects of signal electrode activation might be expected to be proportional to signal intensity, with weak signals generating less activity than strong signals. Onset of the much more intense reinforcing stimulation delivered into the same system should be expected to influence the neural aftereffects of the signals so as to produce a statistical interaction between signal strength and reinforcer strength, with the strongest reinforcers having proportionally more effect on the weakest signals than on the strongest signals.

The fact that there was no indication of an interaction between signal strength and reinforcer strength suggests (a) that the location of the electrodes in contralateral hemispheres precluded interaction, or (b) that the two categories of stimulation (signal and reinforcer) were functionally independent even though all electrodes were in reinforcing loci. Hypothesis (a) seems the less likely alternative, in light of the demonstration by Coons, Schupf, and Ungerleider (1976) that summation of reinforcing stimulation delivered to contralateral sites is possible. However, their reinforcing sites were all within the MFB, while our rats each had one hypothalamic and one septal electrode. The possibility thus remains that bilateral interaction from reinforcing sites occurs only for anatomically symmetrical electrodes.

Even if there were a single reinforcing system upon which all stimulation effects converged, the system might have a sufficiently high threshold to preclude its activation by the relatively weak stimuli used for signal purposes. Since the signals obviously were effective as signals, the fact that no intensity interaction occurred between signals and reinforcers would suggest that signals from reinforcing sites may be processed by a neural system discrete from that which processes the stronger reinforcing stimulation.

There seem to be at least two possible interpretations of the difference in signal detection associated with reinforcer intensity: (1) the most intense reinforcer may have created a higher than optimal level of arousal, thus decreasing the animal's ability to detect the signals; (2) the most intense reinforcer may have caused a shift in the decision criteria used in deciding whether a signal was present on a given trial. The first hypothesis is based on those theories of motivation (e.g., Hebb, 1955) which assume an inverted U-shaped relation between level of arousal and accuracy of performance. Some support for such interpretations can be found in experiments utilizing stimulation of brain stem reticular formation concurrent with presentation of discriminative stimuli, where weak stimulation enhanced and strong stimulation reduced accuracy of discriminative performance (Fuster \& Uyeda, 1962). However, if similar effects had been present in our procedure, we might have expected that the reduction in accuracy of performance in blocks of trials utilizing the most intense reinforcer would have shown approximately equal frequencies of the two types of error (misses and false alarms). Instead, we observed a small, but significant, increase in the proportion of false alarms at the highest reinforcer level. That is, the highest reinforcer intensity produced an increased likelihood of guessing "yes" on trials when the signal was not actually present. 
This result supports the second hypothesis, and is in general agreement with signal detection theory (Swets, 1973), which assumes that such payoff factors as reinforcement may influence the bias of the observer.

The time required to complete each block of 100 trials indicates that the decrease in accuracy of performance at the highest reinforcer value cannot be attributed to response-interference effects of the type which frequently are assumed to account for nonmonotonic rate-intensity curves in self-stimulation procedures (Valenstein, 1964). If such interfering motor effects of the highest reinforcer level had been present, trials using this reinforcer should have required longer times than those using medium or low levels of reinforcement. Instead, the blocks of trials with the medium and high reinforcer values were completed equally quickly and showed no change over the course of the experiment. In contrast, blocks of trials in which the lowest reinforcer level was used showed a significant slowing of speed as the experiment progressed. This observation is in general agreement with other studies in which animals have been repeatedly presented with several reinforcement levels (e.g., Bower, 1961; Keesey, 1962). The fact that intensity of signal had no effect on time to complete blocks of trials indicates that these stimulations were too weak to influence level of motivation, or to serve as "priming"' stimuli (Gallistel, 1969).

These results seem to have several implications for studies of animal psychophysics. For example, the significant effect of reinforcer intensity, with the best absolute levels of detection occurring when the middle intensity of reinforcer was employed, suggests the need for testing with several reinforcer levels if estimates of absolute sensitivity are to be obtained. On the other hand, the absence of a statistical interaction between reinforcer intensity and signal intensity suggests that estimates of relative detectability of signals of various strengths are reasonably independent of intensity of reinforcer used during the testing procedure. In other words, successive determinations of the shape of a psychophysical function should be relatively consistent even though different reinforcer intensities are used for the various replications, while the absolute values of the data points would be expected to show appreciable differences if various reinforcer intensities were utilized.

\section{REFERENCES}

BAss, R. W. Detection of electrical brain stimulation at hypothalamic and septal sites in rats. Journal of Comparative and Physiological Psychology, 1974, 87, 458-465.

BERKLEY, M. A., \& KLING, J. W. A small animal cable coupler with vertical movement compensation. Physiology and Behavior, 1967, 2, 315-316.
BLOUGH, P. M. The visual acuity of the pigeon for distant targets. Journal of the Experimental Analysis of Behavior, 1971, 15, 57-67.

BowER, G. H. A contrast effect in differential conditioning. Journal of Experimental Psychology, 1961, 62, 196-199.

Coons, E. E., Schupf, N., \& Ungerleider, L. G. Uses of double-pulse stimulation behaviorally to infer refractoriness, summation, convergence, and transmitter characteristics of hypothalamic reward systems. Journal of Comparative and Physiological Psychology, 1976, 90, 317-342.

Fuster, J. M., \& UyedA, A. A. Facilitation of tachistoscopic performance by stimulation of midbrain tegmental points in the monkey. Experimental Neurology, 1962, 6, 384-406.

GAllistel, C. R. The incentive of brain-stimulation reward. Journal of Comparative and Physiological Psychology, 1969, 69, 713-721.

GARNER, W. R. The effect of frequency spectrum on temporal integration of energy in the ear. Journal of the Acoustical Society of America, 1947, 19, 808-815.

GerKen, G. M. Electrical stimulation of the subcortical auditory system in behaving cat. Brain Research, 1970, 17, 483-497.

HeBb, D. O. Drives and the CNS (conceptual nervous system). Psychological Review, 1955, 62, 243-254.

Hume, A. L. Auditory detection and optimal response biases. Perception \& Psychophysics, 1974, 15, 425-433. (a)

Hume, A. L. Optimal response biases and the slope of ROC curves as a function of signal intensity, signal probability, and relative payoff. Perception \& Psychophysics, 1974, 16, 377-384. (b)

KEESEY, R. E. The relation between pulse frequency, intensity, and duration and the rate of responding for intracranial stimulation. Journal of Comparative and Physiological Psychology, 1962, 55, 671-678.

KEESEY, R. E. Hypothalamic stimulation as a reinforcer of discrimination learning. Journal of Comparative and Physiological Psychology, 1966, 62, 231-236.

LinDhOLM, E. P., \& KeEsEy, R. E. Discrimination learning as a function of the duration of rewarding hypothalamic stimulation. Psychonomic Science, 1968, 10, 153-154.

KönIG, J. R. R., \& KLIPPEL, R. A. The rat brain. Baltimore: Williams and Wilkins, 1963.

LoNG, C. E. The effects of duration of onset and cessation of light flash on the intensity-time relation in the peripheral retina. Journal of the Optical Society of America, 1951, 41, 743-747.

MAHUT, H. Effects of subcortical electrical stimulation on discrimination learning in cats. Journal of Comparative and Physiological Psychology, 1964, 58, 390-395.

Olds, J., \& Olds, M. Drives, rewards, and the brain. In T. M. Newcomb (Ed.), New directions in psychology II. New York: Holt, Rinehart, \& Winston, 1965.

STEIN, D. G., \& ChOROVER, S. L. Effects of posttrial electrical stimulation of hippocampus and caudate nucleus on maze learning in the rat. Physiology and Behavior, 1968, 3, 787-791.

SwETs, J. The relative operating characteristic in psychology. Science, 1973, 182, 990-1000.

TERMAN, M. Discrimination of auditory intensities by rats. Journal of the Experimental Analysis of Behavior, 1970, 13, 145-160.

Terman, M., \& Kling, J. W. Discrimination of brightness differences by rats with food or brain-stimulation reinforcement. Journal of the Experimental Analysis of Behavior, 1968, 11, 29-37.

Valenstein, E. S. Problems of measurement and interpretation with reinforcing brain stimulation. Psychological Review, 1964, 71, 415-437.

Woodbury, J. W. Action potential: Properties of excitable membranes. In T. C. Ruch \& H. D. Patton (Eds.), Physiology and biophysics. Philadelphia: Saunders, 1965. Pp. 26-58.

Wright, A. A. Psychometric and psychophysical hue discrimination functions for the pigeon. Vision Research, 1972, 12, 1447-1464.

(Received for publication August 2, 1976; accepted August 24, 1976.) 